NISTIR 7986

\title{
LTE Physical Layer Performance Analysis
}

Wen-Bin Yang

Michael Souryal

http://dx.doi.org/10.6028/NIST.IR.7986

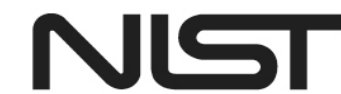

National Institute of Standards and Technology U.S. Department of Commerce 


\title{
LTE Physical Layer Performance Analysis
}

\author{
Wen-Bin Yang \\ Michael Souryal \\ Advanced Network Technologies Division \\ Information Technology Laboratory
}

http://dx.doi.org/10.6028/NIST.IR.7986

May 2014

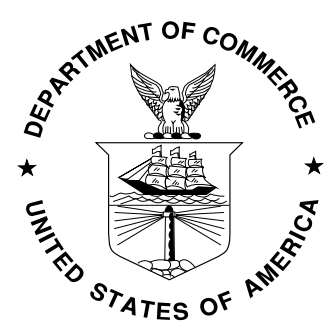

U.S. Department of Commerce Penny Pritzker, Secretary

National Institute of Standards and Technology Patrick D. Gallagher, Under Secretary of Commerce for Standards and Technology and Director 


\title{
LTE Physical Layer Performance Analysis
}

\author{
National Institute of Standards and Technology \\ Wen-Bin Yang and Michael Souryal \\ December 2013
}

\section{Introduction}

The 3GPP Long Term Evolution (LTE) technology exhibits a major advance in wireless communication networks to meet increasing demands for high quality multimedia services. Orthogonal frequency division multiplexing (OFDM) and multiple inputmultiple output (MIMO) are employed to enhance the performance of current wireless systems. With these and other techniques, higher data rates and higher capacity can be attained in the LTE network.

Among these techniques, link adaptation is used in LTE to exploit the frequency selectivity of the wireless channel to improve spectral efficiency. However, to perform link adaptation, certain quality information of the channel is required at the transmitter. During actual network operation, using the channel quality information, link adaption dynamically assigns one of the modulation and coding schemes (MCSs) to each user equipment (UE) in order to maintain link quality. However, for network planning, design or performance evaluation, the performance characteristics (e.g., block error rate as a function of SNR) of each MCS is required.

In this report, we present results of a physical layer performance study for all MCSs (from MCS0 to MCS28) in terms of block error rate (BLER) and spectral efficiency. The results are obtained by using the Steepest Ascent ${ }^{1}$ LTE toolbox for MATLAB which provides functions for modeling the LTE physical layer based upon 3GPP specifications. The performance measures for each MCS are evaluated as a function of signal to noise ratio (SNR), MIMO configuration, and 3GPP multipath fading channel. The 3GPP channels include models for pedestrian, vehicular, and typical urban environments. The results of this study can be used in higher level modeling tools such as cellular network planning and network modeling tools that take abstractions of physical layer performance as input.

\section{LTE Physical Layer}

OFDM technology has been incorporated into LTE because it enables high data rates to be transmitted efficiently while still providing a high degree of resilience to reflections

\footnotetext{
${ }^{1}$ Steepest Ascent Ltd. was acquired by The Mathworks, Inc. in September, 2013.

Certain commercial equipment, instruments, or materials are identified in this report in order to specify the experimental procedure adequately. Such identification is not intended to imply recommendation or endorsement by the National Institute of Standards and Technology, nor is it intended to imply that the materials or equipment identified are necessarily the best available for the purpose.
} 
and interference. The access schemes differ between the uplink and downlink: OFDMA (Orthogonal Frequency Division Multiple) Access is used in the downlink, while SCFDMA (Single Carrier - Frequency Division Multiple Access) is used in the uplink.

The LTE air interface exploits both time division duplex (TDD) and frequency division duplex (FDD) modes to support unpaired and paired spectra. MIMO may also be optionally deployed in the eNodeB and/or UE in order to enhance link quality and to increase data rates/throughputs. MIMO utilizes multiple antennas at the transmitter and/or receiver in combination with adaptive signal processing to provide smart array processing, diversity combining or spatial multiplexing capability. A generic frame structure for the downlink and uplink is shown in Figure 1.

Since the capabilities of the eNodeB and UE are quite different, the LTE PHY downlink and uplink likewise differ and are documented separately in the 3GPP technical specifications. The following two sub-sections briefly describe the technical specifications of the downlink and uplink PHY layers.

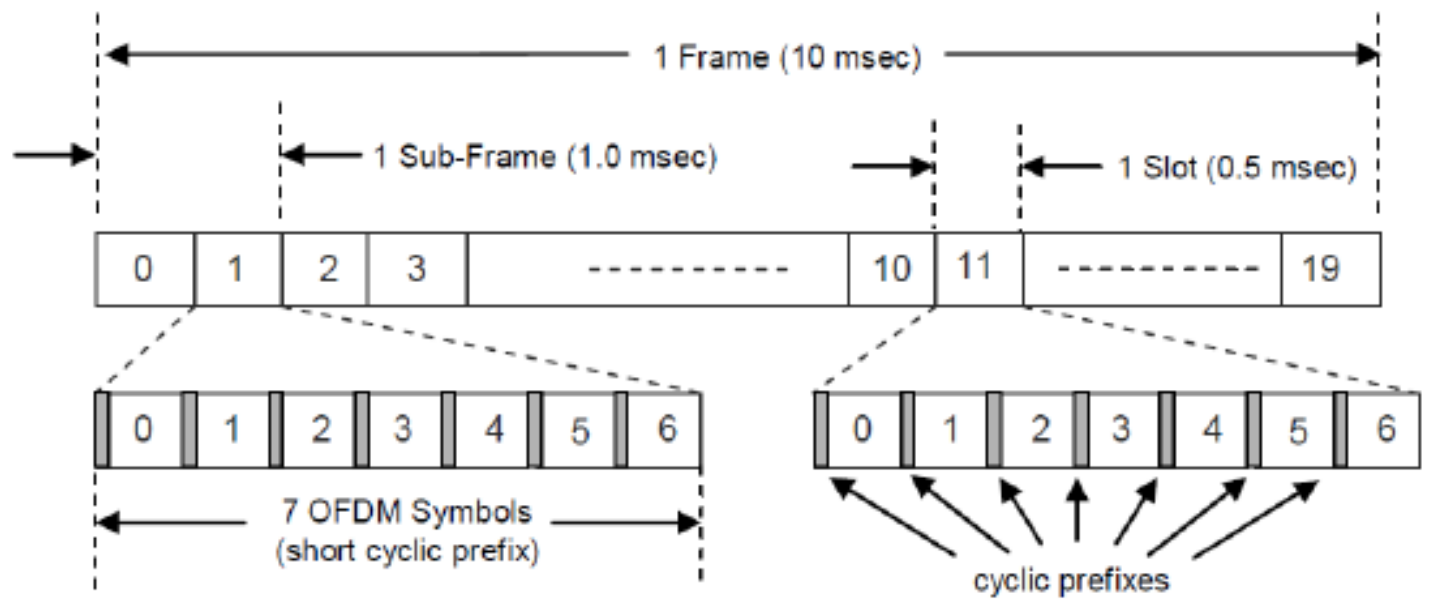

Figure 1: LTE Generic Frame Structure [3]

\subsection{Downlink physical layer}

The LTE downlink PHY is specified for bandwidths from $1.25 \mathrm{MHz}$ to $20 \mathrm{MHz}$. OFDM with a cyclic prefix is the basic modulation scheme selected to combat severe frequencyselective multipath fading. Three different types of physical channels are defined for the LTE downlink: physical downlink shared channel (PDSCH), physical downlink control channel (PDCCH) and common control physical channel (CCPCH). In this report, the focus is on PDSCH performance because it carries user data and is designed for a wide range of data rates. Therefore, modulations include QPSK, 16QAM and 64QAM and the coding rate ranges from $1 / 6$ to $3 / 4$. The downlink uses the generic frame structure shown in Fig. 1 in the time domain. In the frequency domain, it uses a subcarrier spacing of $15 \mathrm{kHz}$. Time-frequency resources are allocated in units of physical resource blocks (PRBs) comprised of 12 subcarriers in frequency and 1 sub-frame in time. The detailed downlink OFDM modulation parameters may be found in [1]. The block diagram of an OFDM transceiver is shown in Figure 2. 


\subsection{Uplink physical layer}

The basic transmission scheme for the LTE uplink is Single-Carrier-Frequency Domain Multiple Access (SC-FDMA). The major advantage of SC-FDMA over conventional OFDM is an approximately $2 \mathrm{~dB}$ lower PAPR (peak-to-average power ratio). In FDD applications, the uplink uses the same generic frame structure as the downlink. It also uses the same subcarrier spacing of $15 \mathrm{kHz}$ and PRB width of 12 subcarriers. Again, only physical uplink shared channel (PUSCH) performance is evaluated here. The PUSCH employs QPSK, 16QAM or 64QAM modulation. The detailed uplink specifications may be found in [8].

The block diagram of the SC-FDMA scheme is also shown in Figure 2. This figure also indicates the difference between OFDMA and SC-FDMA.

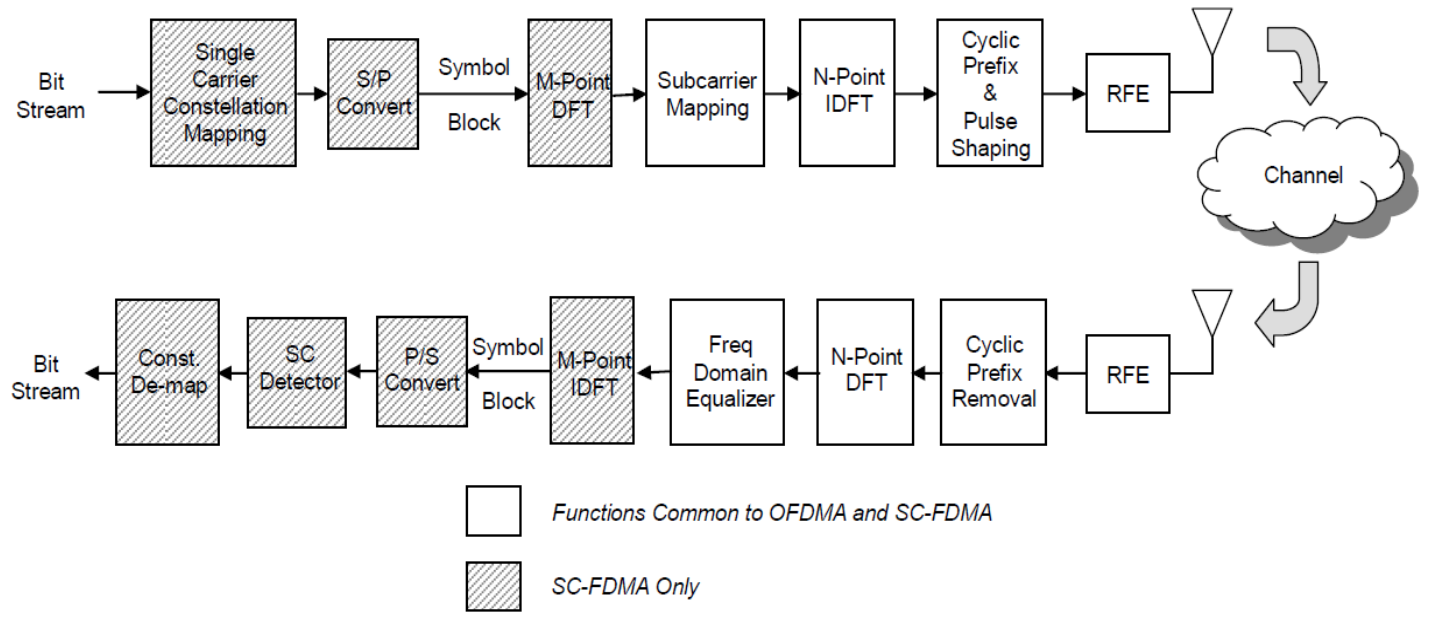

Figure 2: SC-FDMA and OFDMA signal block diagram [3] (S/P = Serial-to-Parallel, DFT = Discrete Fourier Transform, IDFT = Inverse DFT, RFE = RF Front End)

\section{Schemes and fading channels for Performance Analysis}

In this section, MCS and MIMO schemes as well as multipath fading channels and channel estimation are addressed.

\subsection{Modulation and coding scheme}

The modulation and coding scheme (MCS) index value summarizes the modulation type and the coding rate that is used in a given PRB. Typically, a higher MCS index offers a higher spectral efficiency (which translates to a higher potential data rate) but requires a higher SNR to support it. Using our simulation results of BLER vs. SNR, the appropriate MCS index value for a particular channel condition can be determined. 
Annex A of 3GPP technical specifications of Refs. [1] and [8] describes downlink and uplink fixed reference channels, respectively, which may be used for modeling any modulation and coding scheme (MCS) index in terms of number of allocated resource blocks, modulation (QPSK, 16QAM or 64QAM), code rate, and associated transport block size (TBS). Parameters for any MCS index for PDSCH or PUSCH may be obtained by using Tables 7.1.7.1-1, 8.6.1-1 and 7.1.7.2.1-1 [2]. The three tables are listed in Appendix A of this report. Tables A1 and A2 list MCS index vs. TBS index for PDSCH and PUSCH, respectively. Table A3 (portion of Table 7.1.7.2.1-1) shows transport block size as a function of allocated block size and TBS index.

\subsection{MIMO}

One of the main problems of previous telecommunications systems is that of multiple signals arising from the many reflections that are encountered, which may cause interference with one another. By using MIMO, these additional signal paths can be used to their advantage and can increase the throughput.

When using MIMO, it is necessary to use multiple antennas to enable the different paths to be distinguished. While it is relatively easy to add more antennas to a base station, the same is not true of mobile handsets, where the dimensions of the user equipment limit the number of antennas to be placed at least a half wavelength apart. A 1x2 MIMO configuration for uplink and 2x2 for downlink are studied. For the downlink, both transmit diversity and spatial multiplexing are considered in this study.

\subsubsection{Transmit diversity}

The transmit diversity scheme [6] relies on the use of multiple antennas at the transmitter in combination with pre-coding in order to achieve spatial diversity when transmitting a single data stream. Usually transmit diversity necessitates the absolute channel information at the transmitter, but it becomes feasible to implement transmit diversity without the knowledge of the channel with space time block coding [4]. The simplest of the diversity techniques is the Alamouti space time coding scheme [5]. The use of transmit diversity is common in the downlink of cellular systems because it is cheaper and easier to install multiple antennas at the base station than to put multiple antennas on every handheld device. In transmit diversity, to combat instantaneous fading and to achieve considerable gain in instantaneous SNR, the receiver is being provided with multiple copies of the transmitted signal. Hence, applying transmit diversity creates extended converge and better link quality even when users experience poor channel conditions.

\subsubsection{Spatial Multiplexing}


Spatial multiplexing [6] can provide a substantial increase in data rates by transmitting different data streams over parallel channels provided by the multiple transmit and receive antennas, while using the same bandwidth and with no additional power expenditure. In MIMO systems, the increase in capacity is linearly related to the number of transmit/receive antenna pairs. Consider a MIMO system with $M$ transmit and $N$ receive antennas; the radio channel for this system will consist of $M \times N$ ideally uncorrelated paths. This configuration offers $\min (M, N)$ parallel channels that permit simultaneous transmission of data streams. In a $2 \times 2$ system, for example, a single bit stream is split into two half-rate bit streams, modulated and transmitted simultaneously from both antennas which can cause interference to each other at the receiver. Therefore at the receiver, inference cancellation is employed to separate the different transmitted signals. For the spatial multiplexing technique, several decoding algorithms are developed for interference cancellation for the narrowband frequency flat fading case. The spatial multiplexing may be either open-loop or closed-loop MIMO schemes. Openloop spatial multiplexing uses large cyclic delay diversity to pursue maximum multiplexing gain. Closed-loop spatial multiplexing uses pre-coding feedback from the UE to improve SNR or capacity.

\subsection{GPP multipath fading channels}

The multipath fading channel is defined by a combination of a multipath delay profile and a maximum Doppler frequency, which can be 5, 70 or $300 \mathrm{~Hz}$. Annex B of 3GPP technical specification [1] describes the multipath fading propagation conditions, including taps of the Extended Pedestrian A model (EPA), Extended Vehicular A model (EVA) and Extended Typical Urban model (ETU) (also shown in Table 1 below) as well as classical Doppler spectrum. In this report, we focus on performance analysis for EPA5, ETU70 and EVA70 models.

Table 1: 3GPP multipath fading channel models [1]

\begin{tabular}{|c|c|c|c|c|c|}
\hline \multicolumn{2}{|c|}{ EPA } & \multicolumn{2}{c|}{ ETU } & \multicolumn{2}{c|}{ EVA } \\
\hline $\begin{array}{c}\text { Excess tap } \\
\text { delay [ns] }\end{array}$ & $\begin{array}{c}\text { Relative } \\
\text { power } \\
{[\mathrm{dB}]}\end{array}$ & $\begin{array}{c}\text { Excess tap } \\
\text { delay [ns] }\end{array}$ & $\begin{array}{c}\text { Relative } \\
\text { power } \\
\text { [dB] }\end{array}$ & $\begin{array}{c}\text { Excess tap } \\
\text { delay [ns] }\end{array}$ & $\begin{array}{c}\text { Relative } \\
\text { power } \\
\text { [dB] }\end{array}$ \\
\hline 0 & 0.0 & 0 & 0.0 & 0 & -1.0 \\
\hline 30 & -1.0 & 30 & -1.5 & 50 & -1.0 \\
\hline 70 & -2.0 & 150 & -1.4 & 120 & -1.0 \\
\hline 90 & -3.0 & 310 & -3.6 & 200 & 0.0 \\
\hline 110 & -8.0 & 370 & -0.6 & 230 & 0.0 \\
\hline 190 & -17.2 & 710 & -9.1 & 500 & 0.0 \\
\hline 410 & -20.8 & 1090 & -7.0 & 1600 & -3.0 \\
\hline & & 1730 & -12.0 & 2300 & -5.0 \\
\hline & & 2510 & -16.9 & 5000 & -7.0 \\
\hline
\end{tabular}




\subsection{Channel estimation}

A radio propagation channel is time-varying depending upon the propagation environment and Doppler effects. Channel estimation is a vital part of a receiver's design as it is used to estimate the time-varying channel condition in wireless systems. As long as the time-varying radio propagation channel can be kept track of, the receiver can recover precise transmitted information. To facilitate the estimation of channel characteristics, LTE uses cell specific reference signals (pilot symbols). The pilot symbols provide an estimate of the channel at a given location within a sub-frame [7]. Through interpolation, it is possible to estimate the channel across an arbitrary number of sub-frames. Implementation of channel estimation and interpolation depends on window sizes in time and in frequency domains. Each hardware vendor may implement the channel estimator in its own way. Therefore, to minimize the ambiguity of the channel estimation implementation, we assume a perfect channel estimator is employed in our simulations. The effect of practical estimators can be incorporated by shifting the performance curves accordingly (e.g., $1 \mathrm{~dB}$ ).

\section{Simulation Results}

In this study, we assume 5 PRBs are allocated to the link. Although an allocation of 5 PRBs is assumed, we did not see a significant difference in BLER performance when varying the number of allocated PRBs from 1 to 50. The multipath fading channels include EPA5, ETU70 and EVA70. ${ }^{2}$ MIMO configurations are $2 \times 2$ and $1 \times 2$ for downlink and uplink, respectively. The LTE downlink employs OFDMA multiplexing schemes including transmit diversity and spatial multiplexing. FDD and hybrid automatic repeat request (HARQ) are used in the simulations. PDSCH and PUSCH BLER-vs.-SNR curves are shown in the following subsections. Each figure contains BLER curves for all MCSs. Also, a combined spectral efficiency curve is included that is based upon the maximum spectral efficiency over all MCSs at any SNR value.

\subsection{Downlink PDSCH performance using transmit diversity scheme}

\subsubsection{Block error rate}

Figures 3-5 show downlink PDSCH block error rate vs. SNR for all MCSs (MCS0 MCS28) using transmit diversity over EPA5, ETU70 and EVA70 channels, respectively. As expected, lower MCS index values result in lower BLER than higher index values (at the expense of lower spectral efficiency).

\footnotetext{
${ }^{2}$ Because of the longer coherence time of the EPA5 channel model, statistically significant results with this channel model requires the simulation of substantially more frames than with the other channel models.
} 


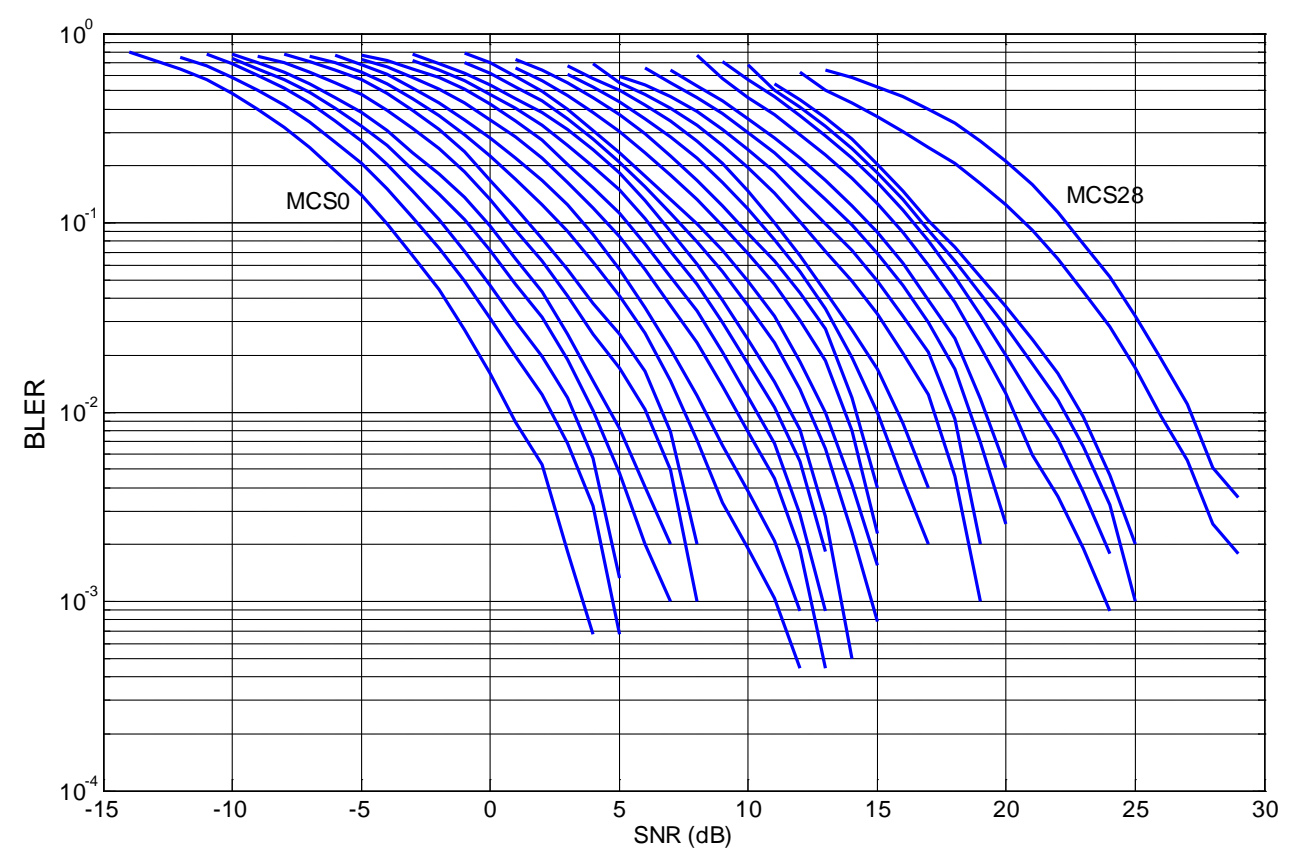

Figure 3: Downlink transmit diversity block error rate over EPA5 channel for all MCSs

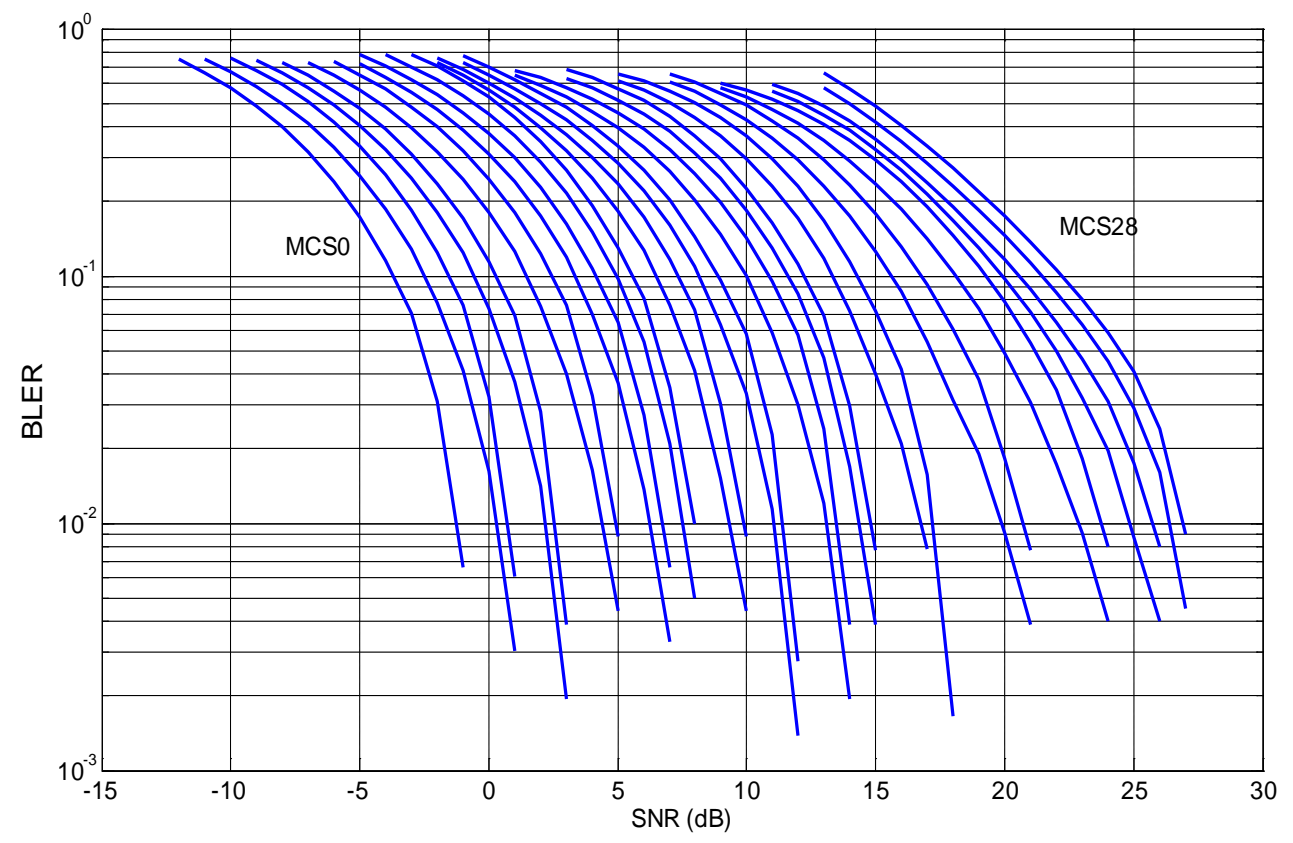

Figure 4: Downlink transmit diversity block error rate over ETU70 channel for all MCSs 


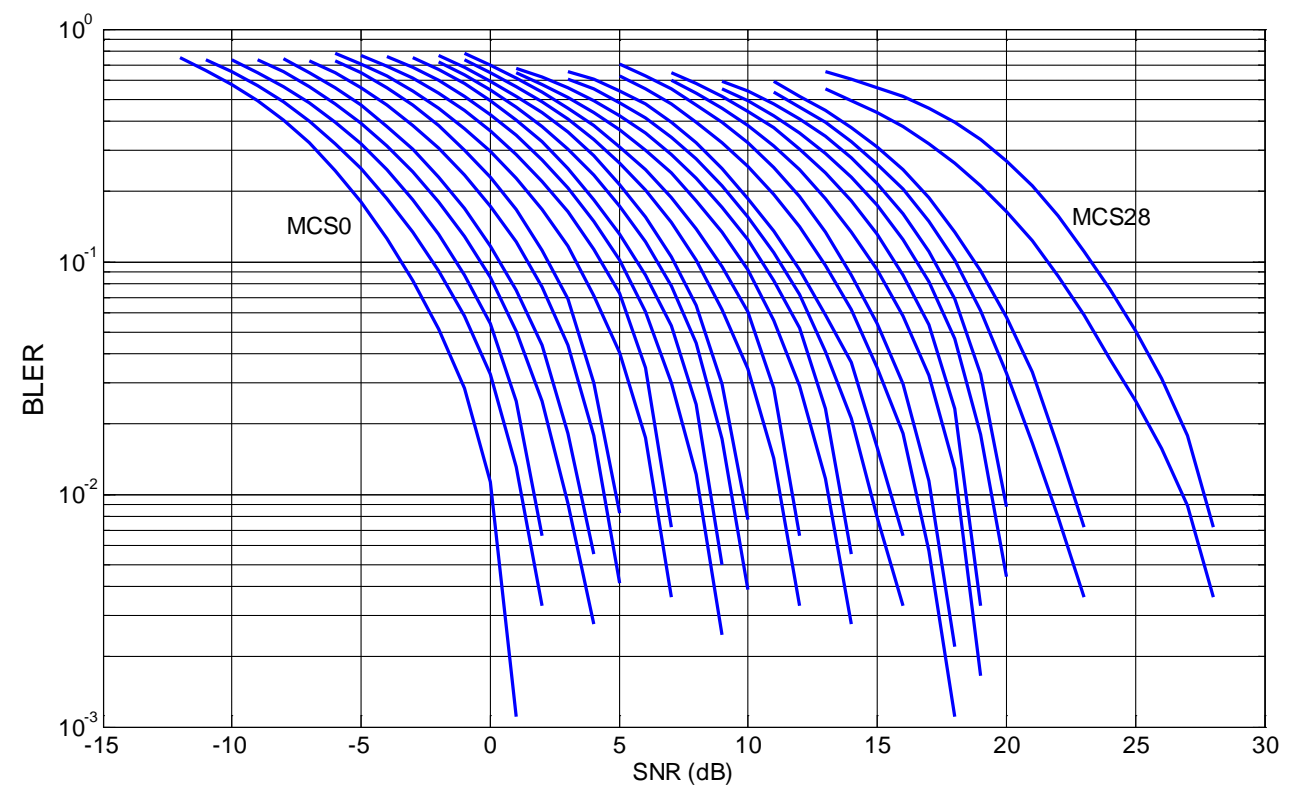

Figure 5: Downlink transmit diversity block error rate over EVA70 channel for all MCSs

\subsubsection{Spectral efficiency}

Figure 6 shows spectral efficiency when transmit diversity is employed. There is no significant difference among the three fading channels except in the range of 10-23dB of SNR.

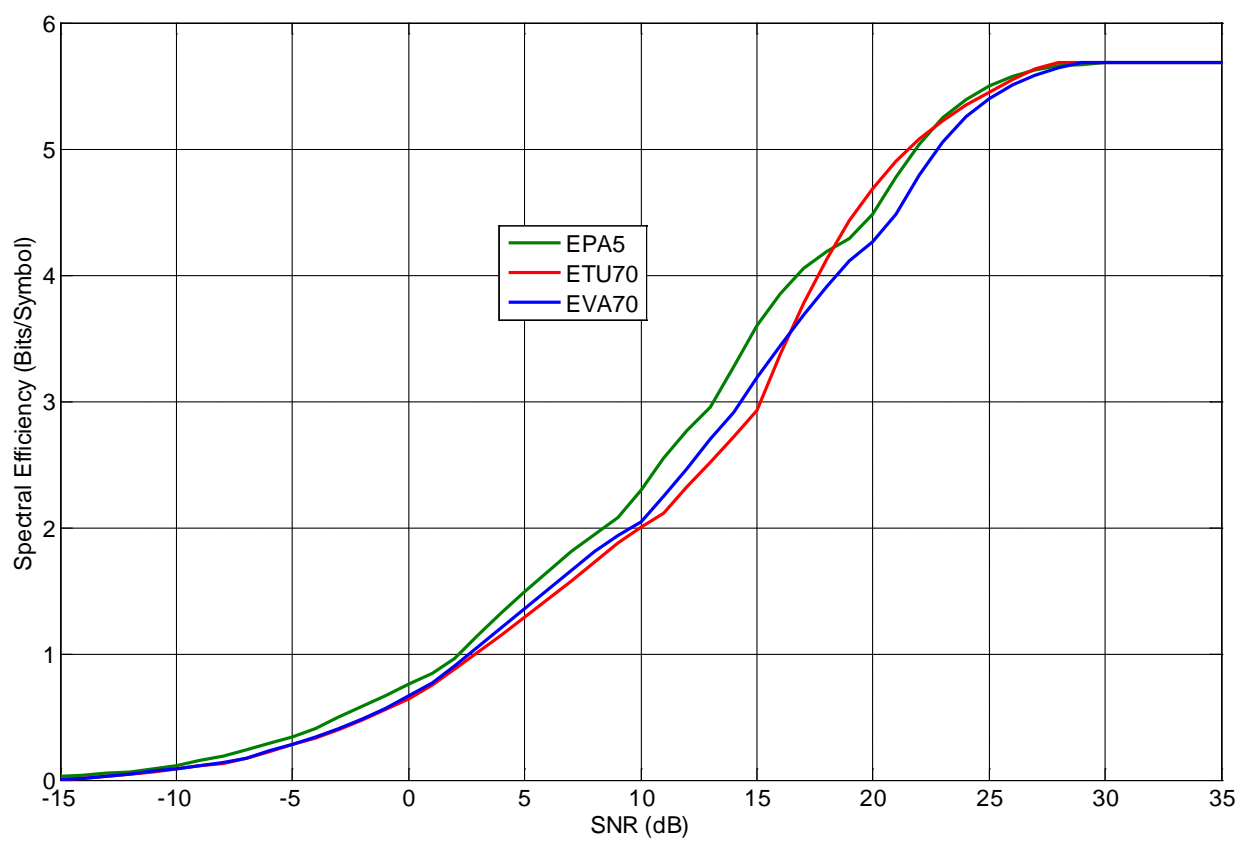

Figure 6: Downlink spectral efficiency using transmit diversity scheme 


\subsection{Downlink PDSCH performance using open-loop spatial multiplexing scheme}

\subsubsection{Block error rate}

Figures 7-9 show downlink PDSCH block error rate vs. SNR over EPA5, ETU70 and EVA70 channels, respectively, when open-loop spatial multiplexing scheme is employed.

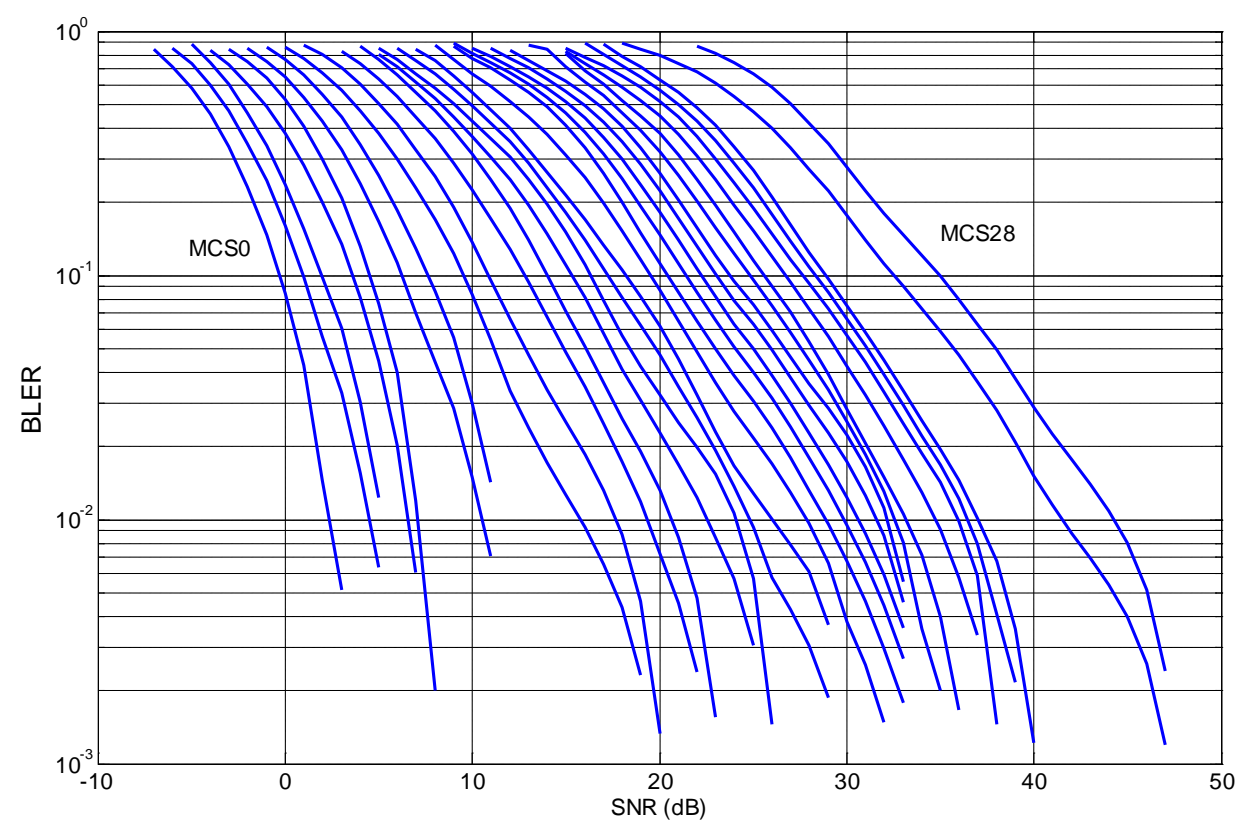

Figure 7: Downlink spatial multiplexing block error rate over EPA5 channel for all MCSs

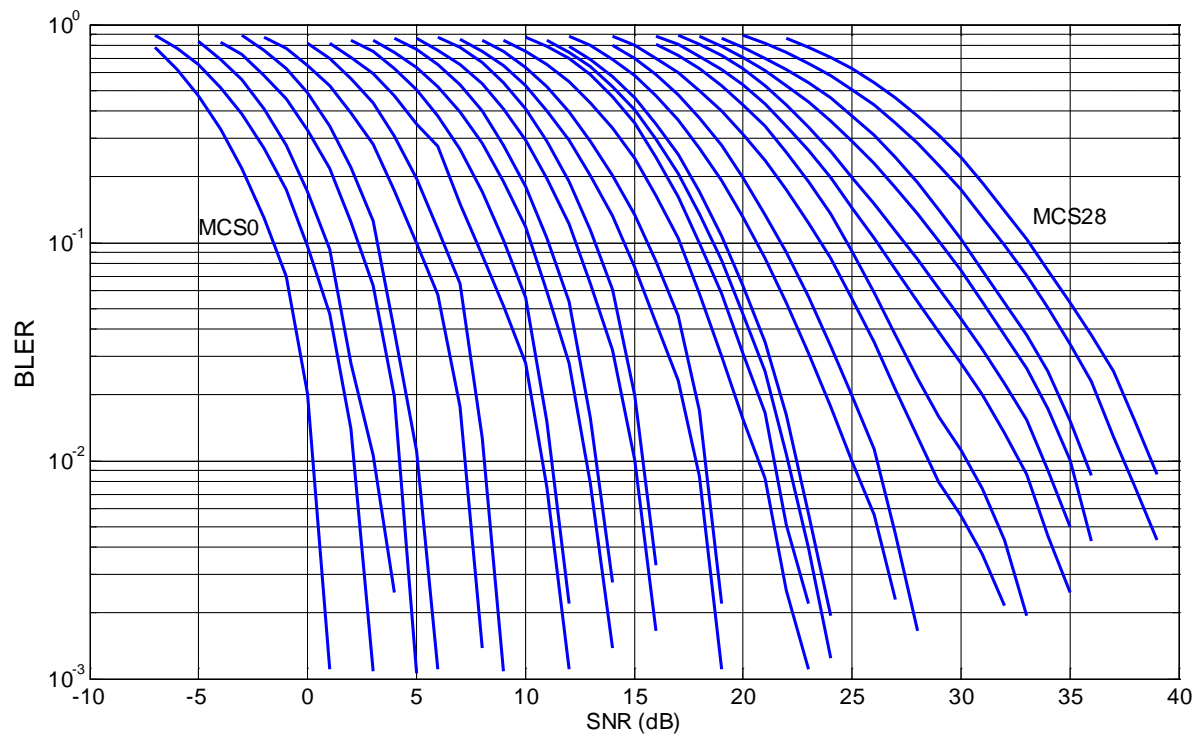

Figure 8: Downlink spatial multiplexing block error rate over ETU70 channel for all MCSs 


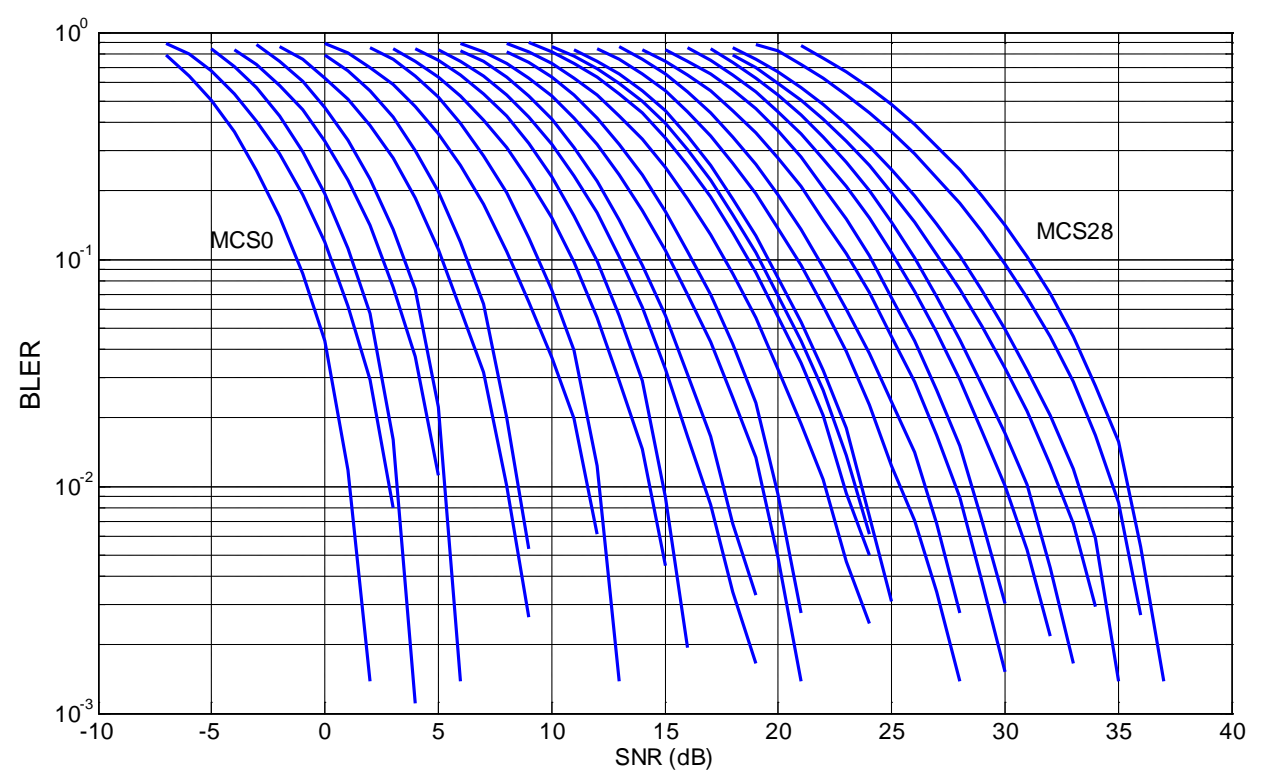

Figure 9: Downlink spatial multiplexing block error rate over EVA70 channel for all MCSs

\subsubsection{Spectral efficiency}

Spectral efficiency curves for the three fading channels are shown in Fig. 10 when openloop spatial multiplexing is employed. In contrast to transmit diversity, spatial multiplexing shows spectral efficiency differentiation in the higher SNR range of 25$40 \mathrm{~dB}$. Furthermore, the peak spectral efficiency is roughly twice that when transmit diversity is employed because of the use of two spatial streams.

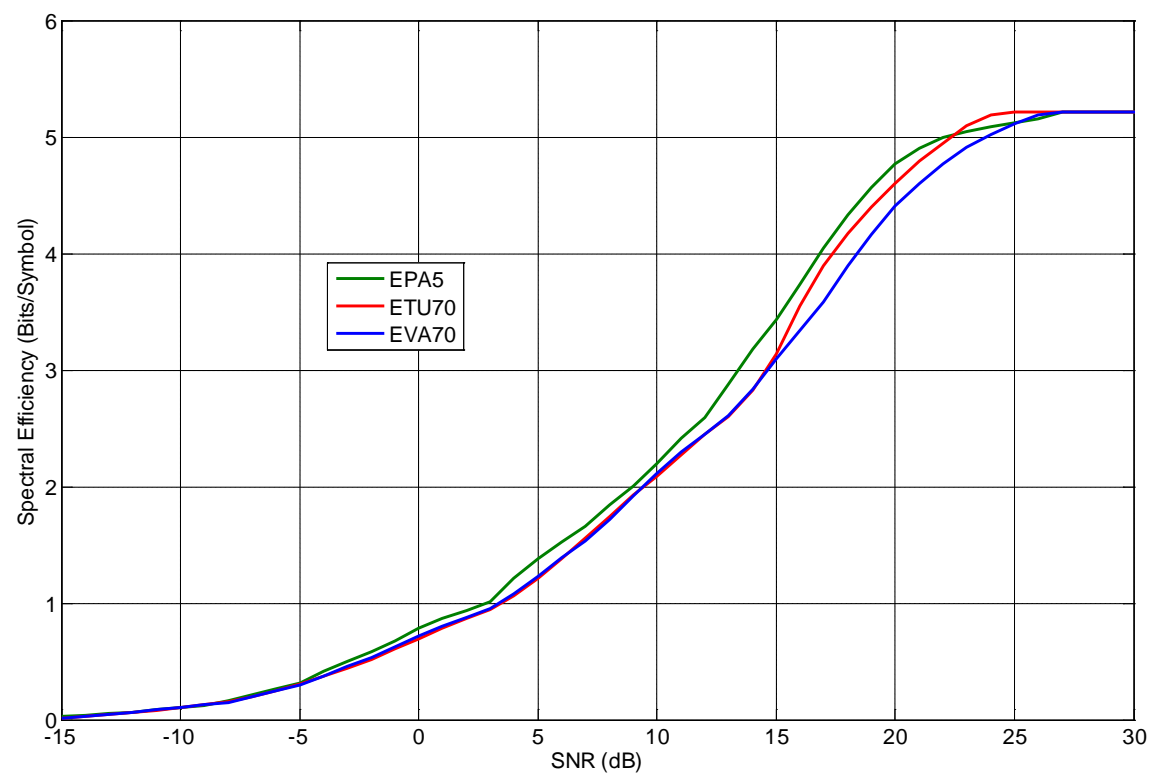

Figure 10: Downlink spectral efficiency using spatial multiplexing scheme 


\subsection{Uplink PUSCH performance}

\subsubsection{Block error rate}

Figures 11-13 show uplink PUSCH block error rate vs. SNR over EPA5, ETU70 and EVA70 channels, respectively.

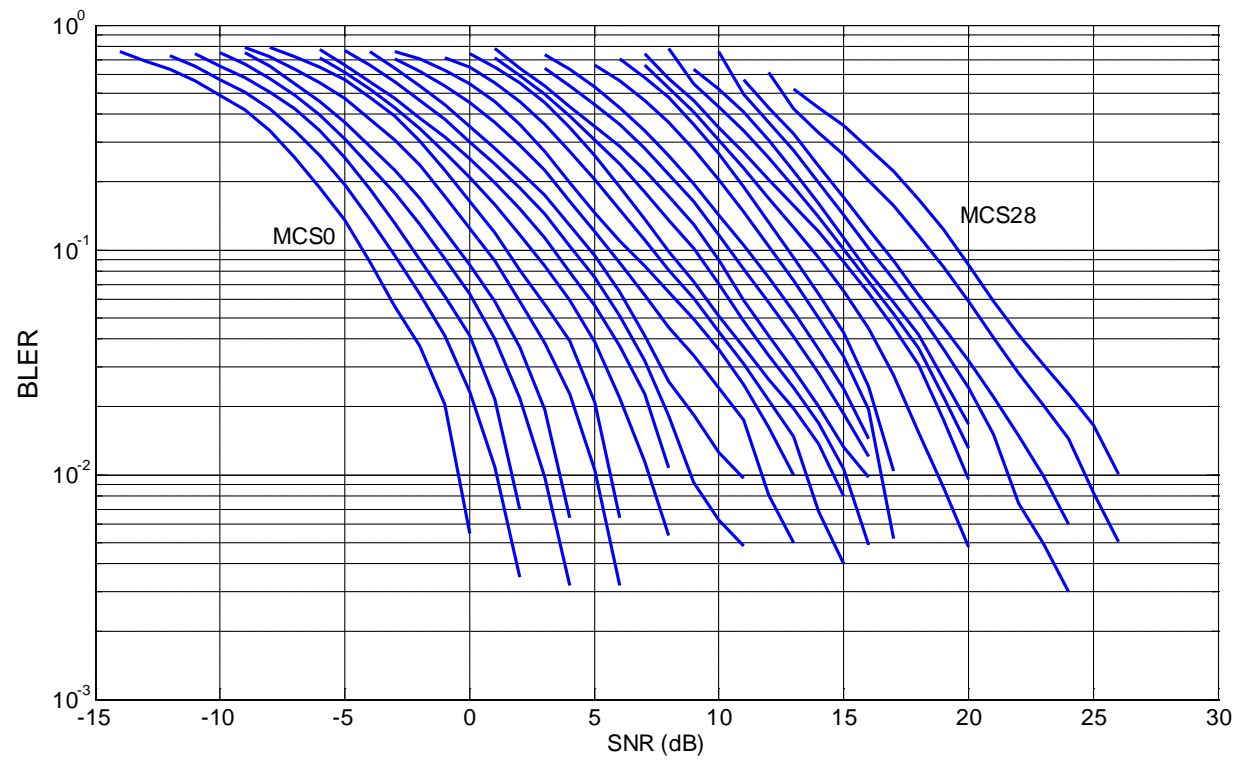

Figure 11: Uplink block error rate over EPA5 channel for all MCSs

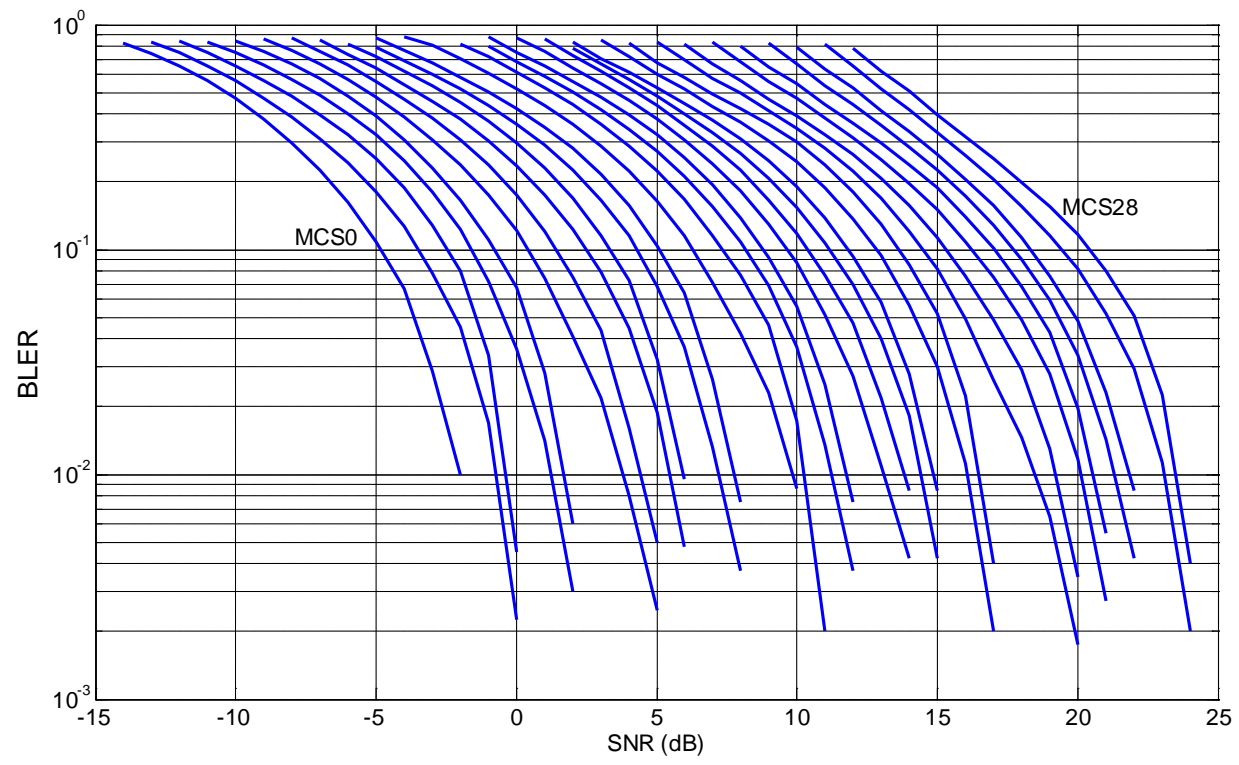

Figure 12: Uplink block error rate over ETU70 channel for all MCSs 


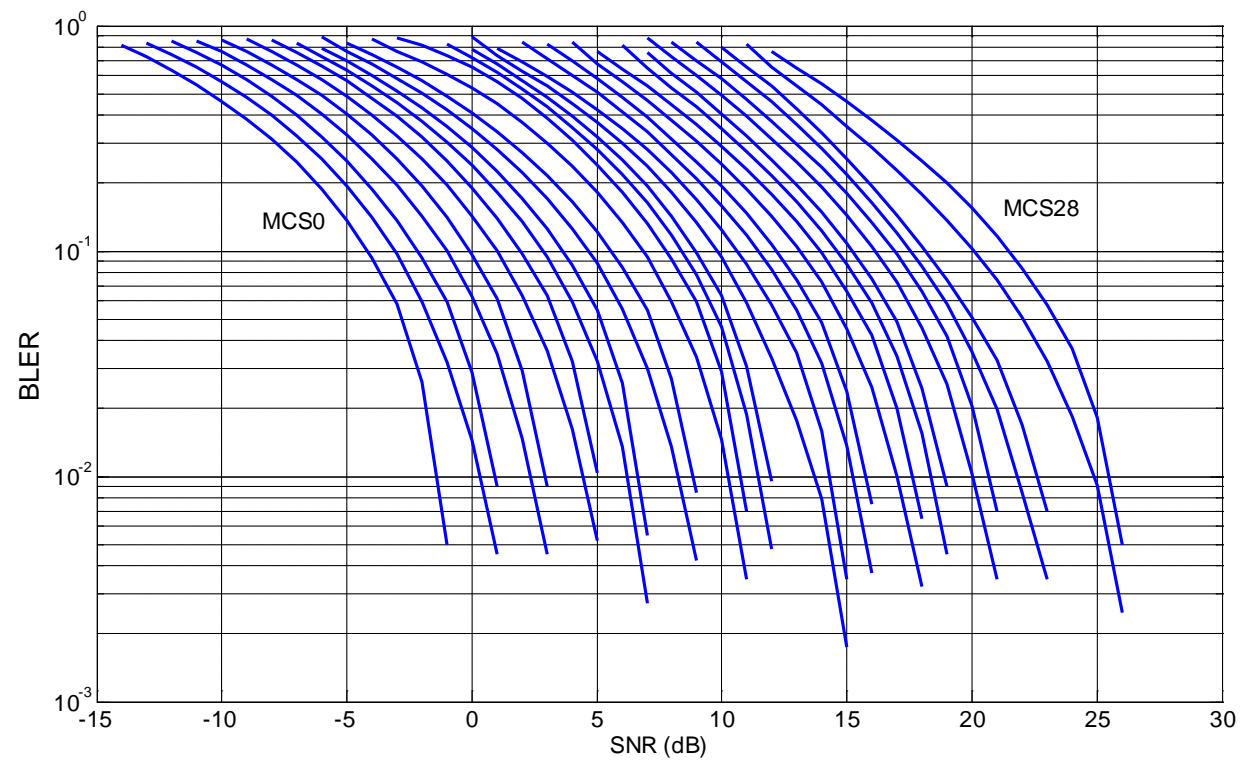

Figure 13: Uplink block error rate over EVA70 channel for all MCSs

\subsubsection{Spectral efficiency}

PUSCH spectral efficiency curves for the three fading channels are shown in Figure 14.

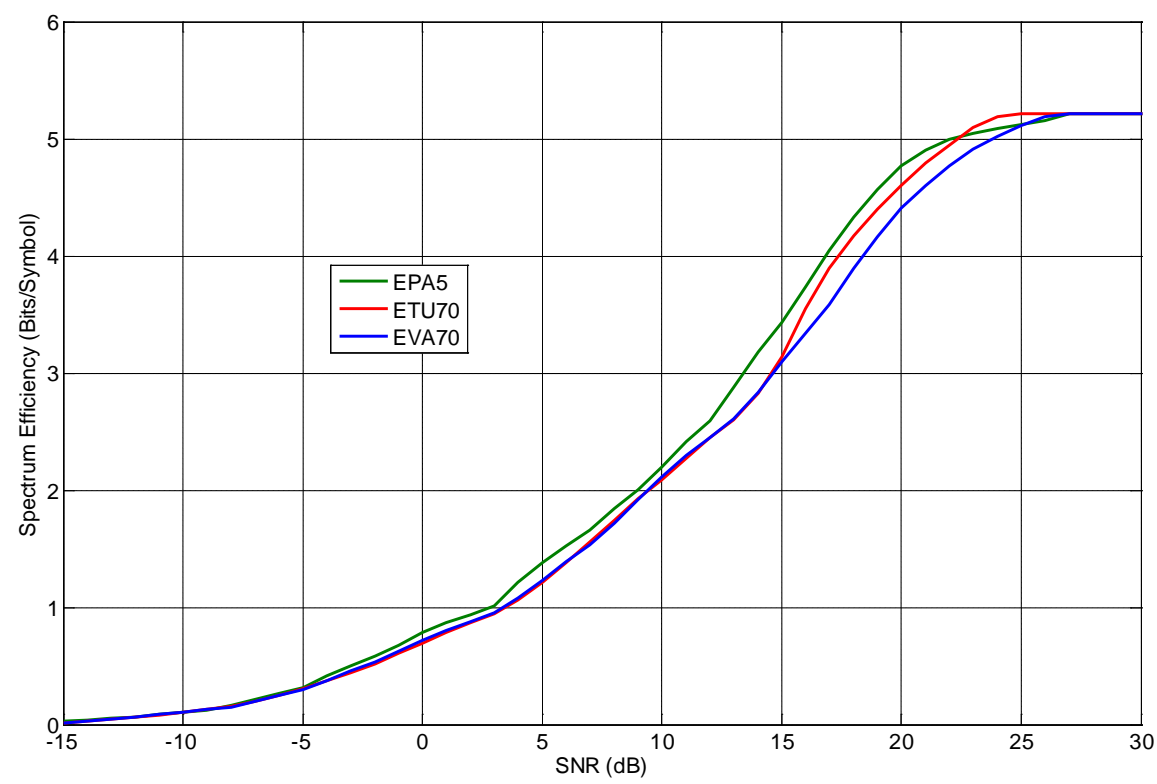

Figure 14: Uplink spectral efficiency 


\section{Summary}

PDSCH and PUSCH performance was evaluated over 3GPP multipath fading channels for all MCS index values (MCS0 to MCS28). The performance measures included block error rate and spectral efficiency. The 3GPP multipath channels were EPA5, ETU70, and EVA70. Transmit diversity and spatial multiplexing schemes for downlink performance were evaluated.

A further simulation study may be performed, if interested, by minor modification from current simulation setups (for example, different fading channel models, different MIMO configurations, or control channel performance study.)

\section{References}

[1] “3GPP TS 36.101 V11.0.0”, March 2012.

[2] “3GPP TS 36.213 V10.5.0”, March 2012.

[3] Jim Zyren, "Overview of the 3GPP Long Term Evolution Physical Layer”, Freescale semiconductor, July 2007.

[4] Mohinder Jankiraman, Space-Time Codes and MIMO Systems, published by Artech House Boston, London, 2004.

[5] S.M. Alamouti, “A Simple Transmit Diversity Technique for Wireless Comm.,’'IEEE Journal Select. Areas Communications, pp. 1451-1458, Vol. 16, No. 8, October 1998.

[6] “3GPP TS 36.211 V11.0.0”, September 2012.

[7] Annex F of “3GPP TS 36.141 V11.0.0”, March 2012.

[8] “3GPP TS 36.104 V11.0.0”, March 2013. 
Appendix A

Table A1: Modulation and TBS index table for PDSCH [2]

\begin{tabular}{|c|c|c|}
\hline $\begin{array}{c}\text { MCS Index } \\
I_{\mathrm{MCS}}\end{array}$ & $\begin{array}{c}\text { Modulation Order } \\
Q_{m}\end{array}$ & $\begin{array}{c}\text { TBS Index } \\
I_{\mathrm{TBS}}\end{array}$ \\
\hline 0 & 2 & 0 \\
\hline 1 & 2 & 1 \\
\hline 2 & 2 & 2 \\
\hline 3 & 2 & 3 \\
\hline 4 & 2 & 4 \\
\hline 5 & 2 & 5 \\
\hline 6 & 2 & 6 \\
\hline 7 & 2 & 7 \\
\hline 8 & 2 & 8 \\
\hline 9 & 2 & 9 \\
\hline 10 & 4 & 9 \\
\hline 11 & 4 & 10 \\
\hline 12 & 4 & 11 \\
\hline 13 & 4 & 12 \\
\hline 14 & 4 & 13 \\
\hline 15 & 4 & 14 \\
\hline 16 & 4 & 15 \\
\hline 17 & 6 & 15 \\
\hline 18 & 6 & 16 \\
\hline 19 & 6 & 17 \\
\hline 20 & 6 & 18 \\
\hline 21 & 6 & 19 \\
\hline 22 & 6 & 20 \\
\hline 23 & 6 & 21 \\
\hline 24 & 6 & 22 \\
\hline 25 & 6 & 23 \\
\hline 26 & 6 & 24 \\
\hline 27 & 6 & 25 \\
\hline 28 & 6 & 26 \\
\hline 29 & 2 & \multirow{3}{*}{ reserved } \\
\hline 30 & 4 & \\
\hline 31 & 6 & \\
\hline
\end{tabular}


Table A2: Modulation, TBS index and redundancy version table for PUSCH [2]

\begin{tabular}{|c|c|c|c|}
\hline $\begin{array}{c}\text { MCS Index } \\
I_{\mathrm{MCS}}\end{array}$ & $\begin{array}{c}\text { Modulation Order } \\
Q_{m}^{\prime}\end{array}$ & $\begin{array}{c}\text { TBS Index } \\
I_{\mathrm{TBS}}\end{array}$ & $\begin{array}{c}\text { Redundancy } \\
\text { Version } \\
r v_{i d x}\end{array}$ \\
\hline 0 & 2 & 0 & 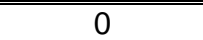 \\
\hline 1 & 2 & 1 & 0 \\
\hline 2 & 2 & 2 & 0 \\
\hline 3 & 2 & 3 & 0 \\
\hline 4 & 2 & 4 & 0 \\
\hline 5 & 2 & 5 & 0 \\
\hline 6 & 2 & 6 & 0 \\
\hline 7 & 2 & 7 & 0 \\
\hline 8 & 2 & 8 & 0 \\
\hline 9 & 2 & 9 & 0 \\
\hline 10 & 2 & 10 & 0 \\
\hline 11 & 4 & 10 & 0 \\
\hline 12 & 4 & 11 & 0 \\
\hline 13 & 4 & 12 & 0 \\
\hline 14 & 4 & 13 & 0 \\
\hline 15 & 4 & 14 & 0 \\
\hline 16 & 4 & 15 & 0 \\
\hline 17 & 4 & 16 & 0 \\
\hline 18 & 4 & 17 & 0 \\
\hline 19 & 4 & 18 & 0 \\
\hline 20 & 4 & 19 & 0 \\
\hline 21 & 6 & 19 & 0 \\
\hline 22 & 6 & 20 & 0 \\
\hline 23 & 6 & 21 & 0 \\
\hline 24 & 6 & 22 & 0 \\
\hline 25 & 6 & 23 & 0 \\
\hline 26 & 6 & 24 & 0 \\
\hline 27 & 6 & 25 & 0 \\
\hline 28 & 6 & 26 & 0 \\
\hline 29 & \multirow{3}{*}{\multicolumn{2}{|c|}{ reserved }} & 1 \\
\hline 30 & & & 2 \\
\hline 31 & & & 3 \\
\hline
\end{tabular}


Table A3: Transport block size table [2]

\begin{tabular}{|c||c|c|c|c|c|c|c|c|c|c|}
\hline \multicolumn{1}{|c||}{} & \multicolumn{10}{c|}{$I_{\text {TBS }}$} \\
\cline { 2 - 11 } & 1 & 2 & 3 & 4 & 5 & 6 & 7 & 8 & 9 & 10 \\
\hline \hline 0 & 16 & 32 & 56 & 88 & 120 & 152 & 176 & 208 & 224 & 256 \\
\hline 1 & 24 & 56 & 88 & 144 & 176 & 208 & 224 & 256 & 328 & 344 \\
\hline 2 & 32 & 72 & 144 & 176 & 208 & 256 & 296 & 328 & 376 & 424 \\
\hline 3 & 40 & 104 & 176 & 208 & 256 & 328 & 392 & 440 & 504 & 568 \\
\hline 4 & 56 & 120 & 208 & 256 & 328 & 408 & 488 & 552 & 632 & 696 \\
\hline 5 & 72 & 144 & 224 & 328 & 424 & 504 & 600 & 680 & 776 & 872 \\
\hline 6 & 88 & 176 & 256 & 392 & 504 & 600 & 712 & 808 & 936 & 1032 \\
\hline 7 & 104 & 224 & 328 & 472 & 584 & 712 & 840 & 968 & 1096 & 1224 \\
\hline 8 & 120 & 256 & 392 & 536 & 680 & 808 & 968 & 1096 & 1256 & 1384 \\
\hline 9 & 136 & 296 & 456 & 616 & 776 & 936 & 1096 & 1256 & 1416 & 1544 \\
\hline 10 & 144 & 328 & 504 & 680 & 872 & 1032 & 1224 & 1384 & 1544 & 1736 \\
\hline 11 & 176 & 376 & 584 & 776 & 1000 & 1192 & 1384 & 1608 & 1800 & 2024 \\
\hline 12 & 208 & 440 & 680 & 904 & 1128 & 1352 & 1608 & 1800 & 2024 & 2280 \\
\hline 13 & 224 & 488 & 744 & 1000 & 1256 & 1544 & 1800 & 2024 & 2280 & 2536 \\
\hline 14 & 256 & 552 & 840 & 1128 & 1416 & 1736 & 1992 & 2280 & 2600 & 2856 \\
\hline 15 & 280 & 600 & 904 & 1224 & 1544 & 1800 & 2152 & 2472 & 2728 & 3112 \\
\hline 16 & 328 & 632 & 968 & 1288 & 1608 & 1928 & 2280 & 2600 & 2984 & 3240 \\
\hline 17 & 336 & 696 & 1064 & 1416 & 1800 & 2152 & 2536 & 2856 & 3240 & 3624 \\
\hline 18 & 376 & 776 & 1160 & 1544 & 1992 & 2344 & 2792 & 3112 & 3624 & 4008 \\
\hline 19 & 408 & 840 & 1288 & 1736 & 2152 & 2600 & 2984 & 3496 & 3880 & 4264 \\
\hline 20 & 440 & 904 & 1384 & 1864 & 2344 & 2792 & 3240 & 3752 & 4136 & 4584 \\
\hline 21 & 488 & 1000 & 1480 & 1992 & 2472 & 2984 & 3496 & 4008 & 4584 & 4968 \\
\hline 22 & 520 & 1064 & 1608 & 2152 & 2664 & 3240 & 3752 & 4264 & 4776 & 5352 \\
\hline 23 & 552 & 1128 & 1736 & 2280 & 2856 & 3496 & 4008 & 4584 & 5160 & 5736 \\
\hline 24 & 584 & 1192 & 1800 & 2408 & 2984 & 3624 & 4264 & 4968 & 5544 & 5992 \\
\hline 25 & 616 & 1256 & 1864 & 2536 & 3112 & 3752 & 4392 & 5160 & 5736 & 6200 \\
\hline 26 & 712 & 1480 & 2216 & 2984 & 3752 & 4392 & 5160 & 5992 & 6712 & 7480 \\
\hline \hline & & & & & & & & & & \\
\hline
\end{tabular}

